diagnosis and AFIP scores to be independently associated with worse prognosis.

Conclusion A combination of surgery and imatinib may be necessary to provide a curative treatment for GISTs and prevent recurrence. Although our study is limited by small numbers, current risk-categorisation models appear to over-estimate recurrence risk with discrepancies in predicting behaviour for certain low-risk tumours. A weighted scoring system combining independent factors associated with poor prognosis may serve as a more accurate clinical prediction tool.

Disclosure of Interest M. Friel: None Declared, Y. Vishwanath: None Declared, H. Wescott: None Declared, N. Wadd: None Declared, A. Dhar Consultant for: Honoraria for advisory board to the Pharmaceutical Industry, Speaker bureau with: Falk Pharma UK, Warner Chilcott UK, Shire Pharmaceuticals, Ferring Pharmaceuticals.

\section{PTU-163 URINARY VOLATILE ORGANIC COMPOUND ANALYSIS TO DISTINGUISH COELIAC DISEASE FROM IRRITABLE BOWEL SYNDROME: A PILOT STUDY}

IJ Covington, ${ }^{2} \mathrm{M}$ McFarlane*, ${ }^{3} \mathrm{R}$ Harbord, 'E Westenbrink, ${ }^{2} \mathrm{~S}$ Chambers, ${ }^{2} \mathrm{~A}$ Dhaliwal, ${ }^{2} \mathrm{~N}$ O'Connell, ${ }^{2} \mathrm{C}$ Bailey, ${ }^{2} \mathrm{C}$ Nwokolo, ${ }^{4} \mathrm{~K}$ Bardhan, ${ }^{2,5} \mathrm{R}$ Arasaradnam. ${ }^{1}$ School of Engineering, University of Warwick, Coventry, UK; ${ }^{2}$ Gastroenterology, UHCW, University of Warwick, Coventry, UK; ${ }^{3}$ MOAC Doctoral Training Centre, University of Warwick, Coventry, UK; ${ }^{4}$ Gastroenterology, Rotherham General Hospital, Rotherham, UK; ${ }^{5} \mathrm{CSRI}$, University of Warwick, Coventry, UK

\subsection{6/gutjnl-2014-307263.237}

Introduction Coeliac disease (CD), a T-cell-mediated gluten sensitive enteropathy, affects $\sim 1 \%$ of the UK population, and in adults presents with a wide range of clinical features; often mistaken for irritable bowel syndrome (IBS). Heightened clinical awareness and serological screening identifies those likely to have $\mathrm{CD}$; the diagnosis confirmed by histological features in small bowel/duodenal biopsies. Limitations to diagnosis are false negative serology (e.g., in IgA deficient patients, the young and the elderly) and reluctance to undergo biopsy. Examining the pattern of urinary volatiles offers a novel non-invasive approach. The gut microbiome is perturbed in several gastrointestinal disorders, resulting in altered gut fermentation patterns, and recognisable by analysis of volatile organic compounds (VOC) in urine, breath and faeces. The altered structure of the small intestinal mucosa, increased gut permeability and altered gluten peptide metabolism, we hypothesised, would change the

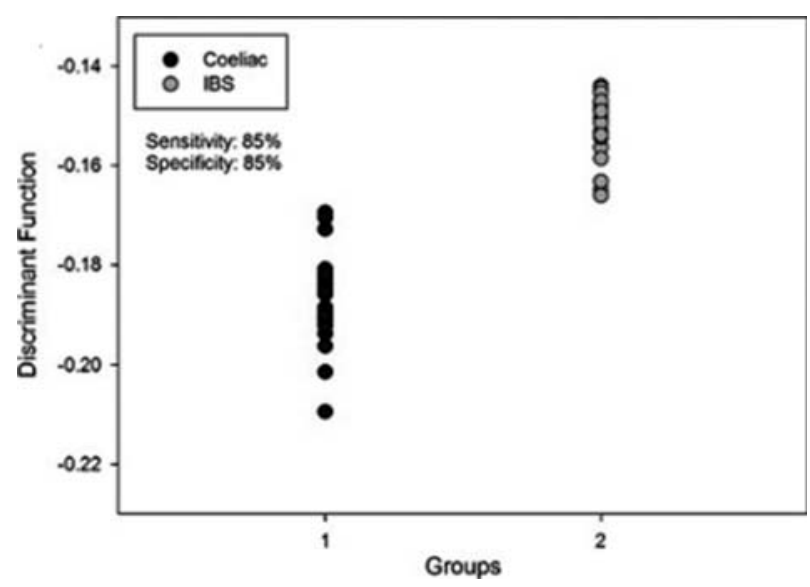

Abstract PTU-163 Figure 1 microbiome creating a unique "fermentome" pattern, distinguishable from IBS. We investigated this by examining the urinary VOC pattern using Field Asymmetric Ion Mobility Spectrometry (FAIMS).

Methods 47 patients were recruited, 27 with $\mathrm{CD}$ and 20 with diarrhoea-predominant IBS (D-IBS). Urine was collected and $10 \mathrm{ml}$ aliquots were stored frozen in universal containers. For assay, the containers were heated to $40 \pm 0.1^{\circ} \mathrm{C}$. The headspace above the sample was then analysed by FAIMS. Linear discriminant analysis (LDA) was used for statistical evaluation.

Results LDA showed that FAIMS distinguishes the VOC pattern in CD vs D-IBS with a sensitivity and specificity of $85 \%$ respectively.

Conclusion This pilot study suggests that FAIMS offers a novel non-invasive approach to identify those likely to have $\mathrm{CD}$, and distinguishes from D-IBS. It may have the potential to non-invasively track the progress of $\mathrm{CD}$ when on a gluten-free diet, to monitor adherence and observe changes.

Disclosure of Interest None Declared.

\section{PTU-164 EVIDENCE OF TWO AETIOLOGIES OF GASTROESOPHAGEAL JUNCTIONAL CANCERS BASED ON GASTRIC PARIETAL CELL DENSITY}

${ }^{1} \mathrm{MH}$ Derakhshan*, ${ }^{2} \mathrm{~T}$ Harvey, ${ }^{2} \mathrm{R}$ Ferrier, ${ }^{1} \mathrm{EV}$ Robertson, ${ }^{2} \mathrm{C}$ Orange, ${ }^{2} \mathrm{M}$ Forshaw, ${ }^{2} \mathrm{JJ}$ Going, ${ }^{1} \mathrm{KE}$ McColl. ${ }^{1}$ Institute of Cardiovascular and Medical Sciences, University of Glasgow, Glasgow, UK; ${ }^{2}$ Institute of Cancer Sciences, University of Glasgow, Glasgow, UK

\subsection{6/gutjnl-2014-307263.238}

Introduction Serum pepsinogen I:II ratio, a surrogate marker of atrophic gastritis, suggests that some adenocarcinomas at the gastroesophageal junction (GOJ) develop on a background of atrophic gastritis, similar to non-cardia gastric cancer, while others arise on a backgrounds of healthy, non-atrophic gastric mucosa similar to oesophageal adenocarcinoma. In this current study, we have directly the background gastric body mucosa in patients with junctional adenocarcinomas compared to oesophageal adenocarcinomas and non-cardia gastric cancers.

Methods 127 gastrectomy and oesophagectomy specimens for adenocarcinoma were identified for which clear topographic description allowed assignment to oesophageal, junctional (including cardia) and gastric non-cardia locations. In these gastric body mucosa specimens, well clear of the tumour margin, parietal cells were immunostained using anti- $\mathrm{H}^{+} / \mathrm{K}^{+}$ATPase. Parietal cell density was counted in 3 to 5 well-oriented fields $\left(1 \mathrm{~mm}^{2}\right.$ each) and expressed as mean parietal cell number per $1 \mathrm{~mm}^{2}$ area. Total mucosal thickness, glandular thickness, intestinal metaplasia, inflammation indicated by polymorphonuclear (PMN) and mononuclear (MN) cells and reactive atypia (RA) were also scored. Non-parametric statistics were used to compare distributions.

Results Ten (8\%) cases lacked well-orientated blocks of body mucosa. The remaining 117 patients included 34 oesophageal, 52 GOJ and 31 non-cardia gastric adenocarcinomas. Median (IQR) parietal cell densities were 836 (173), 602 (389) and 411 (334) per $\mathrm{mm}^{2}$ in gastric mucosa of oesophageal, GOJ and gastric cancers, respectively (all differences $\mathrm{P}<0.001$ ). Using a parietal cell density of $630 / \mathrm{mm}^{2}, 85 \%$ of oesophageal adenocarcinomas had a higher and $84 \%$ of non-cardia gastric cancers had a lower values. With the same cut-off, 50\% of GOJ adenocarcinomas were gastric and remaining was oesophageal in origin.

Glandular mucosa was thicker in patients with GOJ cancer compared to gastric $(0.735$ vs. $0.600, p=0.005)$ and thinner 